\title{
Prevalência de Síndrome Metabólica em pacientes atendidos na Estratégia de Saúde da Família de Barra do Garças, MT
}

\author{
Prevalence of metabolic syndrome among patients attended at the Family Health Strategy of Barra do \\ Garças, MT \\ Gisele Almeida Amaral Fonseca ${ }^{1}$, Leomar Arruda David², Graziele Souza Lira Ferrari ${ }^{3}$, Carlos Kusano \\ Bucalen Ferrari ${ }^{4}$ \\ ${ }^{1}$ Especialista em Farmácia Clínica pela Universidade Católica de Goiás (UCG). Professora Assistente da UNIVAR. \\ ${ }^{2}$ Acadêmica de Enfermagem da UNIVAR. ${ }^{3}$ Acadêmica de Educação Física da Universidade Federal de Mato \\ Grosso (UFMT). ${ }^{4}$ Professor Adjunto, coordenador do BIONUTRE (ICBS/UFMT) e orientador no Programa de Pós- \\ graduação Stricto sensu em Imunologia e Parasitologia Básicas e Aplicadas da UFMT.
}

\begin{abstract}
Resumo
Introdução: A frequência e a morbimortalidade por doenças crônicas não-transmissíveis vem aumentando em diversas regiões do país. Objetivo: Verificar a prevalência de Síndrome Metabólica e possíveis fatores associados em população atendida em unidades da Estratégia de Saúde da Família (ESF). Metodologia: Foram avaliados 96 pacientes adultos de dois ESFs em Barra do Garças (MT). Além da caracterização socioeconômica da amostra, foi aplicado questionário de frequência alimentar e avaliação antropométrica (circunferência da cintura e índice de massa corporal). Após as dosagens bioquímicas (glicemia, colesterol totais e triglicerídeos) foi feita a estimativa da prevalência de caracterização da Síndrome Metabólica utilizando o critério do NCEP/ATPIII (EUA). Resultados: A maioria dos pacientes era do gênero feminino, possuía casa própria, fazia consultas regulares, tinha baixo engajamento em atividades físicas regulares, baixa renda e escolaridade, sendo a maioria idosos ou de meia-idade. Do total, $71 \%$ tinham hipertensão arterial, $52 \%$ hiperglicemia, $19,8 \%$ diabetes mellitus e $42 \%$ foram diagnosticados com Síndrome Metabólica. A renda familiar baixa esteve associada ao maior risco, enquanto que o consumo regular de quatro ou mais xícaras de café esteve inversamente associado ao risco de Síndrome Metabólica, o que não ocorreu com as demais variáveis estudadas. Conclusão: a prevalência de Síndrome Metabólica e suas comorbidades afetam, frequentemente, esta população e demandam melhoria das ações de promoção e atenção primária à saúde.

Palavras-chave: Síndrome metabólica. Hipertensão arterial. Diabetes mellitus. Café. Atenção primária à saúde.

\section{Abstract}

Background: the frequency and morbimortality by chronic non-transmissible diseases have been increased in many regions of the nation. Objective: verify the prevalence of Metabolic Syndrome and possible associated factors among population attended in unities from the Family Health Strategy (ESF). Methodology: 96 adult patients from two ESFs in Barra do Garças (MT) were evaluated. Beyond the socioeconomic characterization of the sample, a food frequency questionaire was applied and the anthropometric evaluation were done (waist circumference and body mass index). After biochemical analysis (glycemia, total cholesterol and tryglycerides) the prevalence of Metabolic Syndrome was estimated according to the NCEP/ATPIII (USA) criteria. Results: the majority of patients belong to female gender, had own house, done regular health consultations, had poor regular physical activities, low income and educational level, with predominance of elderly and middle-aged people. $71 \%$ had arterial hypertension, $52 \%$ hyperglycemia, $19.8 \%$ diabetes mellitus, and $42 \%$ were diagnosed with Metabolic Syndrome. Lower family income was associated to increased risk of Metabolic Syndrome, whereas dietary drinking of four or more coffee cups was inversely associated with Metabolic Syndrome risk which did not occur with other studied variables. Conclusion: the prevalence of Metabolic Syndrome and its comorbidities frequently affected this population and deserve improved health promotion practices in the primary health care.
\end{abstract}

Key-words: Metabolic syndrome. Arterial hypertension. Diabetes mellitus. Primary health care.

\section{INTRODUÇÃO}

A associação da obesidade visceral com hiperglicemia, hipertrigliceridemia, redução da fração do HDL-colesterol e hipertensão arterial caracterizam a síndrome metabólica (SM) que aumenta o risco de calcificação coronariana e carotídea, sendo um fator

Recebido em 29/01/2012; revisado em 30/03/2012.

Correspondência / Correspondence: Carlos K. B. Ferrari. Av. Gov. Jaime Campos, 6390, Distrito Industrial, Barra do Garças, MT. CEP: 78.600-000. E-mail: ferrariphd@yahoo.com.br independente de risco de doença cardíaca coronariana (SAELY et al., 2005). Estudo recente demonstrou que a presença de esteatose hepática em pacientes com SM foi fator preditor da gravidade da calcificação coronária (KNOPFHOLZ et al., 2012).

A prevalência geral de SM no Brasil ainda não totalmente conhecida. Porém, diversos estudos em municipalidades brasileiras têm sugerido que a mesma 
pode ser elevada. Um dos primeiros estudos ocorreu em adultos de 23 a 25 anos em Ribeirão Preto (SP) e reportou prevalências de $4,8 \%$ e $10,7 \%$ para mulheres e homens, respectivamente (BARBIERI et al., 2006). Ao contrário do estudo anterior, um estudo em Cavunge, semi-árido baiano, com adultos de 25 a 87 anos, reportou prevalência de SM maior entre as mulheres $(38,4 \%)$ em relação aos homens $(18,6 \%)$ (OLIVEIRA; SOUZA; LIMA, 2006). No estudo nacional VIGITEL que avaliou 49.276 adultos foi estimada uma prevalência de $14,2 \%$ de SM, sendo ligeiramente maior entre as mulheres (15,2\%) que nos homens $(13,2 \%)$ (SÁ; MOURA, 2010). Entretanto, numa população de meia-idade em Florianópolis (SC), a prevalência de $S M$ foi bem mais elevada (42,9\%) (VANHONI; XAVIER; PIAZZA, 2012). O estudo MONICA-WHO, secção Vitória (ES), observou prevalência de $32,9 \%$ para a SM, tendo pouca diferença entre gêneros (RODRIGUES; BALDO; MILL, 2010).

No estudo VIGITEL os principais fatores de risco para SM observados foram idade avançada, hábitos alimentares inadequados, excesso de peso e sedentarismo (SÁ; MOURA, 2010). Recentemente, autores têm sugerido que o baixo peso ao nascer também poderia ser um fator de risco para a SM (SANTOS; OLIVEIRA, 2011).

Devido à importância da síndrome metabólica, este estudo teve como objetivo estudar a prevalência e fatores associados em pacientes atendidos na Estratégia de Saúde da Família de Barra do Garças, MT.

\section{METODOLOGIA}

\section{Amostra e Aspectos Éticos}

Trata-se de estudo transversal, quantitativo, descritivo-exploratório. A colheita de dados ocorreu no período de Fevereiro a Maio de 2012, por meio de entrevistas, avaliação antropométrica, clínica e exames laboratoriais. Dos 101 pacientes cadastrados nas unidades de Ouro Fino e Piracema da Estratégia de Saúde (ESF) de Barra do Garças, foram incluídos 96 sujeitos $(95,1 \%)$, de ambos os gêneros, com idade acima de 18 anos, atendidos nestas unidades da ESF. Apenas cinco pacientes não quiseram participar do estudo.

Todos os participantes assinaram o termo de consentimento livre esclarecido, tendo a pesquisa sido aprovada pelo Comitê de Ética em Pesquisa (CEP) do Hospital Universitário Júlio Müller (HUJM) (protocolo N761/CEP-HUJM/10).

Aplicação do questionário e dosagens bioquímicas

A aplicação do questionário sobre aspectos socioeconômicos e estilo de vida e a colheita de sangue em jejum foram realizadas nas unidades da ESF. Aproximadamente $3 \mathrm{~mL}$ de sangue foram colhidos em cada tubo a vácuo (Becton Dickinson), um contendo EDTA e outro fluoreto. Os tubos com o sangue foram colocados em uma caixa térmica com gelo, para depois serem levados ao laboratório. O tempo entre a colheita e a centrifugação não excedeu à $2 \mathrm{~h}$. Foram determinadas as concentrações séricas de glicose, colesterol total e triglicerídeos, utilizando o espectrofotômetro Coleman 33-D (USA) e os kits enzimáticos Doles (Brasil).

\section{da cintura \\ Aferição da Pressão Arterial e da circunferência}

A aferição da pressão arterial e da circunferência da cintura foi realizada durante as visitas domiciliares pelo pesquisador acompanhado dos agentes comunitários de saúde.

A verificação da pressão arterial foi executada utilizando o estetoscópio e esfigmomanômetro calibrado, o participante estava em posição confortável (sentado), após o descanso de 5 minutos e o antebraço apoiado. O esfigmomanômetro foi posicionado na parte superior do braço com a borda inferior a 2,5 cm acima do espaço antecubital, o estetoscópio posicionado no espaço antecubital. O manguito foi insuflado até que o manômetro registrasse $20 \mathrm{mmHg}$ acima da pressão sistólica habitual do diabético, a válvula do insuflador foi aberta gradualmente e foi lida no manômetro o ponto onde ouviu-se o primeiro ruído - pressão sistólica, continuou-se a descompressão até o desaparecimento do ruído cardíaco ou abafamento do som - pressão diastólica. Os valores adequados e elevados de pressão arterial seguiram o documento da VI Diretrizes Brasileiras de Hipertensão (SBC, 2010).

A circunferência da cintura $(\mathrm{cm})$ é um indicador antropométrico da adiposidade abdominal. A aferição deste perímetro foi realizada empregando-se uma trena antropométrica (Sanny, modelo SN-4010, EUA), posicionada no ponto médio entre da última costela e a crista ilíaca. Os pacientes foram orientados a ficarem eretos, com o abdome relaxado e com os braços estendidos ao longo do corpo. A leitura da medida foi feita no momento da expiração.

\section{Critério de Síndrome Metabólica}

O critério diagnóstico adotado foram as diretrizes do National Cholesterol Education Program, Adult Panel III (NCEP/ATPIII) (GRUNDY et al., 2004). Caracterizou-se como síndrome metabólica a presença de obesidade abdominal (visceral), mensurada pela circunferência da cintura (e" $88 \mathrm{~cm}$ na mulher; $e^{\prime \prime} 102 \mathrm{~cm}$ no homem), associada a pelo menos mais duas alterações metabólicas: hipertrigliceridemia (TGe"150mg/dL); diminuição do HDL-colesterol (HDL d"40mg/dL no homem; HDL d"50mg/dL na mulher); pressão arterial $(130 \times 85 \mathrm{mmHg})$; e hiperglicemia (glicemia em jejum e"100mg/dL).

\section{Análises estatísticas}

Os dados foram analisados com o auxílio do programa EPI-INFO versão 6.04d, no ambiente DOS, no qual foi realizada a análise estatística. A consistência 
Tabela 1 - Aspectos Sócio-Epidemiológicos da população de adultos de duas unidades da ESF de Barra do Garças, MT, Brasil.

\begin{tabular}{|c|c|c|c|}
\hline Variável & Categorias & $\mathrm{N}$ & $\%$ \\
\hline \multirow[t]{2}{*}{ Gênero } & Feminino & 63 & 65,62 \\
\hline & Masculino & 33 & 34,38 \\
\hline \multirow[t]{4}{*}{ Etnia } & Afrodescendente Branco & 51 & 53,12 \\
\hline & Outro & 38 & 39,58 \\
\hline & Não-respondeu & 2 & 2,09 \\
\hline & & 5 & 5,21 \\
\hline \multirow[t]{5}{*}{ Escolaridade } & Analfabeto & 6 & 6,25 \\
\hline & Fundamental & 57 & 59,37 \\
\hline & Médio & 22 & 22,92 \\
\hline & Superior & 4 & 4,16 \\
\hline & Não-respondeu & 7 & 7,30 \\
\hline \multirow[t]{5}{*}{ Estado civil } & Solteiro & 24 & 25,00 \\
\hline & Casado & 39 & 40,62 \\
\hline & Divorciado & 7 & 7,30 \\
\hline & Outros & 22 & 22,92 \\
\hline & Não-respondeu & 4 & 4,16 \\
\hline \multirow[t]{3}{*}{ Idade } & $\leq 40$ & 7 & 7,29 \\
\hline & $>40 \leq 59$ & 23 & 23,96 \\
\hline & $\geq 60$ & 66 & 68,75 \\
\hline \multirow[t]{3}{*}{ Moradia } & Própria & 82 & 85,42 \\
\hline & Alugada & 6 & 6,25 \\
\hline & Não-respondeu & 8 & 8,33 \\
\hline \multirow[t]{4}{*}{ Renda familiar $^{*}$} & s1 S.M. & 48 & 50 \\
\hline & 21-2 S.M. & 20 & 20,83 \\
\hline & > 2 S.M. & 16 & 16,67 \\
\hline & Não-respondeu & 12 & 12,5 \\
\hline \multirow[t]{2}{*}{ Consultas regulares } & Sim & 71 & 73,96 \\
\hline & Não & 25 & 26,04 \\
\hline \multirow[t]{5}{*}{ Atividades físicas } & Não pratica & 11 & 11,46 \\
\hline & $<1$ vez & 36 & 37,50 \\
\hline & $1-2$ vezes & 20 & 20,83 \\
\hline & 3-4 vezes & 22 & 22,92 \\
\hline & 5 ou mais & 7 & 7,29 \\
\hline \multirow[t]{6}{*}{ Refrigerante } & Não bebe & 49 & 51,04 \\
\hline & $1 \mathrm{vez}$ & 20 & 20,83 \\
\hline & 2 vezes & 3 & 3,12 \\
\hline & 3-4 vezes & 18 & 18,75 \\
\hline & 5 vezes ou mais & 5 & 5,21 \\
\hline & Não-respondeu & 1 & 1,05 \\
\hline \multirow[t]{5}{*}{ Café } & Não bebe & 17 & 17,71 \\
\hline & $1-2$ vezes & 24 & 25,00 \\
\hline & 3 vezes & 37 & 38,54 \\
\hline & 4 ou mais & 16 & 16,67 \\
\hline & Não-respondeu & 2 & 2,08 \\
\hline \multirow[t]{2}{*}{ Hipertensão } & Sim & 68 & 70,83 \\
\hline & Não & 28 & 29,17 \\
\hline \multirow[t]{2}{*}{ Diabetes mellitus } & Sim & 19 & 19,79 \\
\hline & Não & 77 & 80,21 \\
\hline \multirow{2}{*}{ Hiperglicemia } & Sim & 50 & 52,08 \\
\hline & Não & 46 & 47,92 \\
\hline \multirow[t]{2}{*}{ Síndrome metabólica } & Feminino & $29 / 63$ & 46,00 \\
\hline & Masculino & $11 / 33$ & 33,33 \\
\hline
\end{tabular}

Em salários-mínimos $(\mathrm{R} \$ 622,00)$. 
interna foi avaliada através do alfa de Cronbach. 0 critério de $0,70>$ foi criado como prova de satisfatória consistência. A hipótese de associação entre as variáveis foi aceita quando o $p$. valor encontrado foi menor que 0,05 . Foram estimadas as razões de prevalência de diversas entre variáveis e a síndrome metabólica.

\section{RESULTADOS}

Com relação à idade, a mínima foi de 24 e a máxima de 92 anos, com média maior para os homens em relação às mulheres (64 $\pm 13,24 ; 61,76 \pm 14,27$ anos). A renda familiar média dos homens foi também maior que a das mulheres $(\mathrm{R} \$ 1016,41 ; \mathrm{R} \$ 977,82)$. A maioria da população é idosa, vive com um salário-mínimo, tem ensino fundamental completo, é afrodescendente, tem casa própria, faz consultas regulares de saúde, não pratica atividades físicas, sendo hipertensa (71\%). A prevalência de diabetes mellitus clinicamente diagnosticado foi de $20 \%$. Um terço dos homens e quase metade das mulheres apresentaram síndrome metabólica, tendo sido de $41,67 \%$ a prevalência geral desta síndrome.

Outros aspectos sócio-epidemiológicos da população estão representados na Tabela 1.

Ao avaliar as razões de probabilidades das variáveis e sua relação com a síndrome metabólica (Tabela 2), embora tenha havido maior prevalência da doença entre as mulheres (Tabela 1 ), a estimativa de OR mostrou não ter ocorrido diferença estatisticamente significante $(p<0,23)$. Também não houve diferenças estatisticamente significantes entre a síndrome metabólica e as variáveis como idade, etnia, escolaridade, estado civil, consumo de refrigerante e consultas regulares de saúde. Houve uma tendência de

Tabela 2 - Razão de Probabilidade das Variáveis e Possíveis Associações com a Síndrome Metabólica

\begin{tabular}{|c|c|c|c|}
\hline Variável & Odds ratio & IC95\% & $\mathrm{P}$ \\
\hline $\begin{array}{l}\text { Gênero } \\
\text { (feminino x masculino) }\end{array}$ & 1,759 & $0,7096-4,1008$ & 0,2327 \\
\hline $\begin{array}{l}\text { Idade (anos) } \\
(\geq 60 \times<60)\end{array}$ & 0,8571 & $0.3708-1,9812$ & 0,7184 \\
\hline $\begin{array}{l}\text { Etnia } \\
\text { (afro } \times \text { não-afro) }\end{array}$ & 2,0778 & $0.7733-5.5826$ & 0,1470 \\
\hline $\begin{array}{l}\text { Escolaridade } \\
\text { (fundamental x } \\
\text { médio/superior) }\end{array}$ & 1,4910 & $0.5659-3.9287$ & 0,1470 \\
\hline $\begin{array}{l}\text { Estado civil } \\
\text { (casado x não-casado) }\end{array}$ & 0,9376 & $0.4066-2.1621$ & 0,8799 \\
\hline $\begin{array}{l}\text { Moradia } \\
\text { (própria x não-própria) }\end{array}$ & 1.2778 & $0.2433-6.7115$ & 0,7721 \\
\hline $\begin{array}{l}\text { Renda familiar } \\
\leq 1 \mathrm{sm} x>1 \mathrm{sm} \\
>1 \mathrm{sm} x \leq 1 \mathrm{sm}\end{array}$ & $\begin{array}{l}3,3158 \\
0,2011\end{array}$ & $\begin{array}{l}1.3543-8.1184 \\
0.0754-0.5361\end{array}$ & $\begin{array}{l}0,0087^{\circ} \\
0,0013^{\circ}\end{array}$ \\
\hline $\begin{array}{l}\text { Consulta regular } \\
\text { ( } \operatorname{sim} \times \text { não) }\end{array}$ & 1,0976 & $0.4337-2.7775$ & 0,8442 \\
\hline $\begin{array}{l}\text { Atividade física } \\
\text { (não } \times \text { sim) } \\
\text { (não } \times 3-4 \text { vezes) }\end{array}$ & $\begin{array}{l}1,1349 \\
0,6895\end{array}$ & $\begin{array}{l}0,4997-2,5778 \\
0,2682-1,7730\end{array}$ & $\begin{array}{l}0,7624 \\
0,4404\end{array}$ \\
\hline $\begin{array}{l}\text { Refrigerante } \\
\text { (3-4 vezes } x \text { não) }\end{array}$ & 0,9275 & $0,3054-2,8171$ & 0,8944 \\
\hline $\begin{array}{l}\text { Café } \\
\text { (xícaras/dia) } \\
\text { (2-3 xícaras x não) } \\
\text { (4 xícaras } x \text { não) }\end{array}$ & $\begin{array}{l}1,0794 \\
0,0499\end{array}$ & $\begin{array}{l}0,3134-3,7175 \\
0,0115-0,2169\end{array}$ & $\begin{array}{l}0,9037 \\
0,0001^{\circ}\end{array}$ \\
\hline Hipertensão & 7,0313 & $2,2087-22,3831$ & $0,001^{\circ}$ \\
\hline Diabetes & 5,1520 & $1,6621-15,97$ & $0,0045^{\circ}$ \\
\hline Hiperglicemia & 30,00 & $7,9269-113,5381$ & $<0,0001^{\circ}$ \\
\hline
\end{tabular}


menor probabilidade de síndrome metabólica no grupo que praticava mais atividade física que, porém, não foi estatisticamente significante. As associações estatisticamente significantes foram baixa renda familiar e presença de hipertensão, diabetes e hiperglicemia. $O$ consumo de quatro xícaras ou mais de café esteve associado a menor probabilidade de síndrome metabólica.

\section{DISCUSSÃO}

Um estudo em Cavunge, semi-árido baiano, com adultos de 25 a 87 anos, reportou prevalência de SM maior entre as mulheres $(38,4 \%)$ em relação aos homens $(18,6 \%)$ (OLIVEIRA; SOUZA; LIMA, 2006). No estudo nacional VIGITEL a prevalência de SM foi de $14,2 \%$ (SÁ; MOURA, 2010). Entretanto, numa população de meiaidade em Florianópolis ( $S C$ ), a prevalência de $S M$ foi bem mais elevada (42,9\%) (VANHONI; XAVIER; PIAZZA, 2012), corroborando os resultados do presente estudo (41,7\%).

Houve uma tendência de maior prevalência de SM entre as mulheres, que não foi confirmada pela análise estatística. Em outros estudos houve maior prevalência de $S M$ entre as mulheres comparadas aos homens (OLIVEIRA; SOUZA; LIMA, 2006; SÁ; MOURA, 2010; SUN et al., 2012), o que não ocorreu em outros trabalhos (BARBOSA et al., 2010; SILVA; PRATA; CUNHA, 2010; VANHONI; XAVIER; PIAZZA, 2012).

Considerando-se a etnia, houve tendência de maior prevalência entre os afrodescendentes, o que não foi comprovado pela análise estatística. Os resultados deste estudo estão de acordo com o trabalho de Pelegrini et al. (2010) que avaliou homens trabalhadores de uma metalúrgica no distrito federal, não observando diferenças étnicas na prevalência de $\mathrm{SM}$. Ao contrário dos resultados deste estudo, um estudo em Salvador (BA), mostrou maior prevalência de SM entre homens brancos e menor entre negros (BARBOSA et al., 2010). No mesmo estudo, a prevalência de SM foi maior entre as mulheres negras em relação às brancas.

Neste estudo ter baixa renda foi associado com maior chance de SM, o que está de acordo com diversos estudos (LOUCKS et al., 2007; PARK et al., 2012; JORDAN et al., 2012).

No presente estudo, ter diabetes, hiperglicemia e hipertensão aumentaram a probabilidade de SM, o que era esperado, uma vez que estas alterações são componentes essenciais da síndrome (ROJAS-MARTINEZ; AGUILAR-SALINAS; JIMENEZ-CORONA, 2012).

No presente estudo não houve associação entre consumo de refrigerante e síndrome metabólica. Dados do Framingham Heart Study mostraram uma forte associação entre consumo de refrigerantes e risco de sobrepeso, obesidade, SM e alterações metabólicas (DHINGRA et al., 2007).
Há forte evidência que o sedentarismo aumenta o risco de SM (SÁ; MOURA, 2010). Neste estudo, houve uma tendência para associação inversa entre atividade física e SM que não foi comprovada estatisticamente.

Há evidências científicas mostrando que o consumo regular de café reduz o risco de diabetes mellitus tipo 2 (VAN DAM; HU, 2005). Num estudo de casocontrole no Japão, o consumo de quatro xícaras de café esteve inversamente associado ao risco de síndrome metabólica $(O R=0,61)$ (MATSUURA et al., 2012). No presente estudo, o consumo regular da mesma quantidade de café esteve associado a uma redução ainda maior na razão de probabilidades.

A falta de associação entre algumas variáveis (sedentarismo, consumo de refrigerantes, idade) e a síndrome metabólica pode ser pelo menos em parte explicadas pela reduzida população aqui estudada.

\section{CONCLUSÃO}

A prevalência de síndrome metabólica nesta população de adultos foi elevada, sendo positivamente associada com baixa renda, hiperglicemia, diabetes e hipertensão arterial e inversamente associada com o consumo regular de quatro xícaras de café.

\section{REFERÊNCIAS}

BARBIERI, M.A. et al. Health in early adulthood: the contribution of the 1978/1979. Ribeirão Preto birth cohort. Braz. J. Med. Biol. Res., Ribeirão Preto, v.39, p.1041-1055, 2006.

BARBOSA, P.J.B. et al. Influência da cor da pele auto-referida na prevalência da síndrome metabólica numa população urbana do Brasil. Arq. Bras. Cardiol., São Paulo, v.94, n.1, p.34-40, 2010.

DHINGRA, R. et al . Soft drink consumption and risk of developing cardiometabolic risk factors and the metabolic syndrome in middle-aged adults in the community. Circulation, Dallas, v.116, p.480-488, 2007.

GRUNDY, S.M. et al. Definition of metabolic syndrome. Circulation, Dallas, v.109, p.433-438, 2004.

JORDAN, H.T. et al. Metabolic syndrome among adults in New York City, 2004 New York City Health and Nutrition Examination Survey. Prev. Chronic Dis., Atlanta, v.9, p.100-260, 2012. doi: http:// dx.doi.org/10.5888/pcd9.100260.

KNOPFHOLZ, J.; Esteatose hepática e calcificação coronariana em pacientes assintomáticos portadores de síndrome metabólica. Rev. Bras. Clín. Méd., São Paulo, v.10, n.1, p.11-14, 2012.

LOUCKS, E.B. et al.Socioeconomic disparities in metabolic syndrome differ by gender: evidence from NHANES III. Ann. Epidemiol., New York, v.17, n.1, p.19-26, 2007.

MATSUURA, $H$. et al. Relationship between coffee consumption and prevalence of metabolic syndrome among Japanese civil servants. J. Epidemiol., Tokyo, v.22, n.2, p.160-166, 2007.

OLIVEIRA, E.P. de; SOUZA, M.L.A. de; LIMA, M.D.A. de. Prevalência de síndrome metabólica em uma área rural do semi-árido baiano. Arq. Bras. Endocrinol. Metab., São Paulo, v.50, p. 456-465, 2006.

PARK, S-J. et al. Sex differences in the relationship between socioeconomic status and metabolic syndrome: the Korean National Health and Examination Survey. Diabet. Res. Clin. Pract., Boston, v.96, n.3, p.400-406, 2012. 
PELEGRINI, A. et al. Prevalência de síndrome metabólica em homens. Rev. Salud Pública, Bogotá, v.12, n.4, p.635-646, 2010.

RODRIGUES, S.L.; BALDO, M.P.; MILL, J.G. Associação entre a razão cintura-estatura e hipertensão e síndrome metabólica: estudo de base populacional. Arq. Bras. Cardiol., São Paulo, v.95, n.2, p.186-191, 2010

ROJAS-MARTINEZ, R.; AGUILAR-SALINAS, C.A.; JIMENEZ-CORONA, A. Optimal cutoff points for the detection of undiagnosed type 2 diabetes, hypertension and metabolic syndrome in Mexican adults. Salud Pública Méx., México, v.54, n.1, p.13-19, 2012.

SÁ, N.N.B. de; MOURA, E.C. Fatores associados à carga de doenças da síndrome metabólica entre adultos brasileiros. Cad. Saúde Pública, Rio de Janeiro, v.26, n.9, p.1853-1862, 2010.

SAELY, C.H.; The metabolic syndrome, insulin resistance, and cardiovascular risk in diabetic and nondiabetic patients. J. Clin. Endocrinol. Metabol., Stanford, v.90, p.5698-5703, 2005.

SANTOS, S.P. dos; OLIVEIRA, L.M.B. Baixo peso ao nascer e sua relação com obesidade e síndrome metabólica na infância e adolescência. Rev. Ciênc. Méd. Biol., Salvador, v.10, n.3, p.329336, set./ dez. 2011.

SILVA, K.F.; PRATA, A.; CUNHA, D.F. da. Freqüência de síndrome metabólica e padrão de ingestão alimentar de adultos vivendo em uma área rural do Brasil. Rev. Soc. Bras. Med. Trop., Brasília, v.44, n.4, p.425-429, 1998.

SUN, D-L. et al. Metabolic syndrome vs. its components for prediction of cardiovascular mortality: A cohort study in Chinese elderly adults. J. Geriatr. Cardiol., Greenwich, v.9, p.123-129, 2002.

VAN DAM, R.M.; HU, F.B. Coffee consumption and risk of type 2 diabetes: a systematic review. JAMA, Chicago, vol.294, n.1, p.97104, 2005.

VANHONI, L.S.; XAVIER, A.J.; PIAZZA, H.E. Avaliação dos critérios de síndrome metabólica nos pacientes atendidos em ambulatório de ensino médico em Santa Catarina. Rev. Bras. Clín. Méd., São Paulo, v.10, n.2, p.100-105, 2012. 